\title{
Les limites de la décentralisation : le cas du logement
}

The Limits of Decentralisation : the Case of Housing

\section{David Fée}

\section{OpenEdition}

\section{Journals}

Édition électronique

URL : http://journals.openedition.org/rfcb/3451

DOI : $10.4000 /$ rfcb.3451

ISSN : 2429-4373

Éditeur

CRECIB - Centre de recherche et d'études en civilisation britannique

Édition imprimée

Date de publication : 1 avril 2005

ISBN : 2-911580-20-6

ISSN : 0248-9015

Référence électronique

David Fée, «Les limites de la décentralisation : le cas du logement », Revue Française de Civilisation Britannique [En ligne], XIII-2 | 2005, mis en ligne le 01 avril 2005, consulté le 10 avril 2020. URL : http:// journals.openedition.org/rfcb/3451; DOI : https://doi.org/10.4000/rfcb.3451

Ce document a été généré automatiquement le 10 avril 2020.

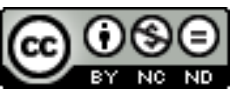

Revue française de civilisation britannique est mis à disposition selon les termes de la licence Creative Commons Attribution - Pas d'Utilisation Commerciale - Pas de Modification 4.0 International. 


\title{
Les limites de la décentralisation : le cas du logement
}

The Limits of Decentralisation : the Case of Housing

\author{
David Fée
}

1 Le New Labour de Tony Blair, on le sait, a été élu sur un ambitieux programme de réforme institutionnelle. Parmi les nombreux chantiers auxquels le gouvernement de Tony Blair s'est attelé depuis 1997, il en est un, moins connu, qui soulève bien des interrogations. Le programme de décentralisation régionale en Angleterre annoncé dans le programme électoral de $1997^{1}$ prévoit la création de chambres régionales dans un premier temps, suivies éventuellement d'assemblées régionales élues. Cette réforme est officiellement justifiée par la nécessité d'accroître la participation citoyenne et de rendre certaines institutions comptables devant les électeurs mais aussi par la volonté $\mathrm{du}$ gouvernement de donner une voix aux régions. Dans le même temps, le gouvernement de Tony Blair a pris la pleine mesure de la crise immobilière que connaît le pays depuis plus d'une décennie et il s'est engagé par la voix du vice-Premier ministre, John Prescott, à y mettre un terme en faisant bâtir un nombre accru de nouveaux logements. Or, ces deux axes politiques se rencontrent en un point hautement sensible qui est celui de la détermination des besoins régionaux en logements. Certains signes laissent penser que ces deux priorités s'avèrent beaucoup plus difficiles à concilier que le gouvernement ne l'avait imaginé et qu'il souhaiterait le laisser croire. Comme j'aimerais le montrer, le langage de la participation citoyenne et de la décentralisation semble mal s'accommoder d'un climat de pénurie immobilière.

\section{Une situation critique}

2 Le gouvernement de Tony Blair a hérité d'une situation immobilière préoccupante, caractérisée par la pénurie et donc l'inflation, dont ses prédécesseurs conservateurs ne sont pas entièrement responsables. Le Livre vert de 1977, Housing Policy: A Consultative Document ${ }^{2}$, le dernier à être publié sur le logement avant l'année $2000^{3}$, soulignait déjà qu'il existait un déséquilibre entre rythme de construction et besoins en logements. $\mathrm{Ce}$ 
diagnostic a été confirmé au début des années 1980 par plusieurs associations, qui toutes s'accordaient à dire que seul un vigoureux programme de construction jusqu'en l'an 2000 pouvait compenser le déficit annuel en logements et venir à bout du problème des mal-logés ${ }^{4}$. Or, les politiques de logement des conservateurs n'ont rien fait pour pallier la pénurie mais bien au contraire l'ont aggravée, et ce pour plusieurs raisons.

3 Tout d'abord, le volume des dépenses consacrées par le gouvernement au logement public a été réduit de $53 \%$ de 1979 à $1992^{5}$, ce qui a conduit à un effondrement spectaculaire de la construction publique. Alors que les collectivités locales bâtissaient 74000 logements en 1980, elles n'en construisaient plus que 1369 en $1995^{6}$. En outre, conformément aux promesses contenues dans le programme conservateur de 1979, 1,7 millions de HLM ont été vendus à leur propriétaire de 1979 à 1997 grâce au Right to Buy inclus dans le Housing Act de 1980 et les recettes de ces ventes ont été immobilisées et n'ont pas permis de compenser ces pertes. Qui plus est, les perspectives de retour à un équilibre ont été compliquées par l'évolution démographique britannique: les prévisions du DoE quant au nombre de nouveaux foyers ont été revues à la hausse en $1995^{7}$ et portées à 4,4 millions entre 1991 et 2016, soit près d'un million de plus que les prévisions précédentes.

4 Paradoxalement, malgré cette aggravation de la pénurie, le logement ne semble pas avoir été considéré comme une priorité électorale par le Parti travailliste avant son élection. Ainsi en 1996, dans New Britain, Tony Blair consacrait-il un simple chapitre au logement, chapitre dans lequel le futur Premier ministre s'engageait avant tout à accroître la propriété ainsi qu'à permettre aux municipalités d'user de leurs recettes ${ }^{8}$. Le manifeste de 1997 n'était guère plus alarmiste et reprenait globalement les mêmes objectifs, insistant seulement sur le fait que 'Capital receipts from the sale of council houses received but not spent by local councils will be reinvested in building new houses and rehabilitating old ones. ${ }^{9}$ En accord avec ces engagements, le gouvernement nouvellement élu a annoncé dans un premier temps le déblocage de 800 millions de livres; en juillet 1998, au vu des conclusions de la Comprehensive Spending Review, 3,9 milliards supplémentaires de livres ont été consacrés sur trois ans par le chancelier de l'Échiquier à la rénovation des logements dans le cadre de la régénération urbaine et à la construction de logements en milieu urbain sur d'anciens terrains industriels ${ }^{10}$.

\section{Un bilan décevant}

5 Quatre ans plus tard, à la veille d'une nouvelle élection et en dépit de cette manne financière, le gouvernement n'a pu que constater l'inutilité de ses efforts. Dans un marché du logement désormais marqué par l'absence de l'État, la solution ne peut venir que du secteur privé car le secteur associatif ne dispose pas des moyens nécessaires. Or, depuis 1998, les constructeurs privés bâtissent en moyenne 140000 logements par an ${ }^{11}$ et la baisse de la construction de 12,5\% enregistrée par le secteur privé depuis 1993 n'a pas été enrayée ${ }^{12}$. Pis encore, en 2002, 163000 logements au total ont été construits au Royaume Uni, soit moins qu'à aucun autre moment depuis $1939^{13}$. Enfin, le nombre de logements sociaux bâtis n'a jamais été aussi bas, avec 24000 constructions en 2002, alors qu'il en faudrait au minimum 43000 selon le propre aveu du gouvernement ${ }^{14}$. Ces données inquiétantes restent d'actualité. Elles viennent en effet d'être confirmées par l'enquête réalisée pour le compte du gouvernement par Kate Barker de la Banque d'Angleterre, qui a conclu à la nécessité de bâtir un minimum de 39000 logements 
supplémentaires afin de suivre la courbe démographique, voire 93000 à 146000 logements supplémentaires afin de retrouver les conditions prévalant dans les années $1980^{15}$

Devant ce constat d'échec John Prescott a donné le coup d'envoi en 2003 à un ambitieux programme ${ }^{16}$ dénommé Sustainable Communities: Building for the Future. Celui-ci doit en particulier permettre la construction de 200000 logements supplémentaires d'ici 2006 dans quatre zones de croissance choisies dans l'est et le sud-est du pays. Surtout, le gouvernement a infléchi sa politique. Il semble désormais convaincu que le système d'urbanisme et d'aménagement du territoire hérité du Town and Country Planning Act de 1947 est responsable de la pénurie et présente la réforme du système comme la clef du problème ${ }^{17}$. Ce système aujourd'hui fortement critiqué par le gouvernement pour sa lenteur, son inefficacité et sa complexité et comme ne permettant pas une véritable participation citoyenne ${ }^{18}$ trouve son origine dans la volonté des gouvernements d'après-guerre d'obliger les collectivités locales à maîtriser l'urbanisation. Les bases du système ont été jetées en 1947 et modifiées en 1968 par une seconde loi. Ce système impose aux collectivités locales responsables ${ }^{19}$ de dresser un cadastre de leur territoire et de préparer un plan d'aménagement ${ }^{20}$. Ce plan peut être plus au moins détaillé selon la nature de la collectivité. Celui des comtés (structure plan), doit indiquer sur 10/15 ans les principes et projets généraux d'occupation future des sols, et ventiler le nombre de nouveaux logements par districts. Pour ceux-ci, le plan (local plan) doit préciser la nature et l'implantation des constructions. Fait nouveau pour l'après-guerre, ce système de plan ne crée pas en lui-même un droit de construire et la collectivité peut exercer un droit de veto sur tout projet de construction.

7 C'est ce système que le gouvernement entend réformer, comme il l'a expliqué dans le Livre vert de 2001, Planning: Delivering a Fundamental Change, en le simplifiant. Il envisage de remplacer les deux niveaux de plans par un plan unique (Local Development Framework) qui comprendrait une déclaration (statement of core policies) dessinant les objectifs à long terme d'aménagement et de régénération, la stratégie choisie, les critères retenus pour contrôler l'aménagement. Le Local Development Plan devrait aussi expliquer comment la population locale serait associée, par le biais d'une charte (statement of Community involvement) et fournir un plan d'action plus détaillé (action plan) identifiant les priorités à court terme dans les zones de changement (telles que l'emplacement des nouveaux logements par exemple), ainsi qu'une carte montrant les zones de changement ${ }^{21}$.

Le gouvernement semble donc déterminé à hâter et faciliter la construction du plus grand nombre de logements possible, y compris en recourant à la menace de sanctions financières comme l'ont encore récemment montré les déclarations de John Prescott à l'encontre de certaines collectivités du sud accusées de rechigner à mettre sur le marché de nouveaux terrains ${ }^{22}$. Or cette volonté de relancer le bâtiment et d'accroître le parc immobilier semble mal s'articuler avec le programme de décentralisation du New Labour.

\section{Le programme régional du gouvernement}

Comme le souligne le Livre blanc consacré aux régions publié en 2002, Your Region, Your Choice, le gouvernement de Tony Blair entend officiellement présider à une renaissance régionale pour trois raisons. 
10 La première raison est d'ordre politique. La décentralisation est en effet censée réduire le déficit démocratique, rendre le gouvernement local comptable et offrir aux citoyens la possibilité de s'exprimer sur les décisions locales ${ }^{23}$. Le Livre blanc rejoint en cela l'objectif d'améliorer la démocratie locale défini dès 1998 dans le Livre blanc Modern Government in Touch with People. Cela passe, selon le gouvernement, par une meilleure "gouvernance locale ». Il faut entendre par cela la traduction des objectifs stratégiques gouvernementaux au niveau local au travers de la collaboration et du partenariat d'organisations locales gouvernementales et non gouvernementales. Le deuxième motif au programme régional du gouvernement est d'ordre économique car la décentralisation est censée décupler le potentiel économique des régions en permettant aux acteurs locaux et à la population d'élaborer des stratégies adaptées aux problèmes et aux besoins régionaux ${ }^{24}$. Elle doit permettre de libérer l'énergie des régions et donc de bâtir une nation plus prospère en facilitant l'emploi et la production. Enfin, la régionalisation obéit à un troisième objectif, stratégique cette fois, qui est de permettre une meilleure coordination de la gestion des problèmes, qui parfois dépassent le cadre des collectivités locales actuelles, à savoir celui du district ou du comté.

11 Concrètement, l'avancée de la décentralisation au niveau régional a été rythmée par trois étapes. La première s'est traduite sur le terrain par la création en 1999 de huit nouvelles institutions, les Regional Development Agencies ${ }^{25}$. Celles-ci ont reçu la mission de renforcer l'économie régionale, à savoir promouvoir le développement économique durable, la régénération de leur région ainsi que la compétitivité et l'emploi ${ }^{26}$. Leur composition est dominée par les partenaires économiques locaux, le secteur associatif, mais doit inclure $30 \%$ au moins d'élus locaux ${ }^{27}$. Cette première phase a aussi été marquée par l'établissement, sur une base volontaire, de huit Regional Chambers, à partir de 1998. Ces institutions, auto-proclamées assemblées, sont composées pour $70 \%$ d'élus locaux et pour $30 \%$ d'acteurs sociaux, économiques et environnementaux de la région et ont un double rôle. Exécutif tout d'abord, car elles contrôlent l'activité des $R D A$ et ont un droit de regard sur leur regional economic strategy; stratégique enfin, car elles sont responsables de la coordination des diverses stratégies régionales et du développement de partenariats avec les organismes privés, associatifs et publics ou semi-publics.

12 Dans un second temps le gouvernement a cherché à renforcer les institutions régionales pré-existantes, en particulier les neuf Government offices for the Regions créés en 1994 par les conservateurs pour être «les yeux et les oreilles du gouvernement central " dans les régions et traduire localement les politiques des neuf ministères parrains ${ }^{28}$. Depuis 2000 , les $G O$ ont donc reçu de nouvelles responsabilités qui doivent leur permettre d'assurer une meilleure adéquation des mesures gouvernementales au contexte local. Elles doivent désormais en particulier coordonner de nouveaux programmes gouvernementaux, collaborer avec les huit Regional Development Agencies et contrôler leur activité. Cette période a également été marquée par la création de l'Autorité du Grand Londres en 2000.

13 Enfin, une troisième étape s'est ouverte après la réélection du parti travailliste avec la publication en 2002 du Livre blanc Your Region, Your Choice consacré aux régions. Ce document concrétise l'engagement ${ }^{29}$ pris par le gouvernement dès 1997 de permettre aux électeurs régionaux de se prononcer par référendum sur la création d'assemblées 
régionales directement élues qui bénéficieront d'un transfert important de responsabilités exécutives et stratégiques.

Dans le cadre de cette stratégie de régionalisation, le gouvernement a donc indiqué dès 1998 sa volonté d'accroître ce qu'il nomme la regional ownership ${ }^{30}$ des politiques de logement, à savoir permettre aux régions d'élaborer des politiques de construction qui répondent à la situation locale grâce à l'implication de tous les acteurs locaux. Ceci passe par la réforme du processus de calcul des besoins en logements régionaux, jugé trop centralisateur par le gouvernement au profit d'un partenariat entre acteurs locaux, et donc d'un plus fort degré de gouvernance locale ; l'objectif affiché est de conférer au processus une légitimité accrue (greater legitimacy ${ }^{31}$ ) selon les termes du ministre.

15 Jusqu'en 1997, les politiques régionales de logement étaient le résultat d'une cascade de chiffres du centre vers la périphérie. Les organismes régionaux d'aménagement (regional planning bodies), à savoir des conférences régionales des représentants des collectivités territoriales, se fondaient sur les estimations démographiques ${ }^{32} \mathrm{du}$ Office of National Statistics pour calculer les besoins régionaux en logements ${ }^{33}$. Elles transmettaient leurs propositions aux Government offices, qui étaient susceptibles de réclamer une modification si ces chiffres ne cadraient pas avec la politique nationale de logement formulée depuis 1988 dans la directive d'aménagement n³ (Planning Policy Guidance 3). Une fois cette modification acceptée ou imposée, une directive régionale (Regional Planning Guidance) était émise officiellement par le ministre de tutelle, qui précisait le nombre de logements requis en moyenne par an pour les 15/20 ans à venir et répartissait ces logements entre comtés ou districts métropolitains. Le ministre avait donc le dernier mot dans l'élaboration des directives ${ }^{34}$. De même, le gouvernement central contrôlait la politique régionale de logement social par le biais de la formulation d'un Regional Housing Statement devant identifier les priorités régionales en matière d'investissement dans le parc social existant. Ce rapport était élaboré par le Government Office de la région et la Housing Corporation, "quango " responsable du financement des bailleurs sociaux. Le système de formulation des politiques de logement jusqu'en 1997 accordait donc une faible latitude aux collectivités territoriales car il préservait une hiérarchie (a top-down approach) permettant au gouvernement central d'asseoir son autorité ${ }^{35}$.

Le gouvernement propose donc de lui substituer un autre système qui transférerait 'responsibility away from central governement to the bodies with a regional stake in its implementation ${ }^{36}$. Depuis le vote du Planning and Compulsory Purchase Act en 2004, les huit assemblées régionales sont les organismes responsables de la planification régionale et, à la différence des anciennes conférences régionales d'aménagement, ne sont plus cantonnées à un rôle de conseillers. Elles doivent formuler, en collaboration avec les $R D A$ et le GO, une ébauche de directive régionale ${ }^{37}$, désormais dénommée Regional Spatial Strategy. Puis au terme d'un processus de navette entre l'assemblée et le ministère, celui-ci publie la directive finale qui a à présent force de loi. Autre exemple de gouvernance locale affiché haut et fort par le gouvernement, chaque région doit se doter d'un Regional Housing Board qui doit formuler une Regional Housing Strategy, en d'autres termes évaluer la situation locale et formuler une stratégie ainsi que des recommandations au ministre de tutelle en matière d'investissement pour le logement social régional ${ }^{38}$. 
17 La prochaine étape du programme de régionalisation doit permettre aux assemblées régionales élues de formuler et publier une Regional Spatial Strategy incluant le logement, sans que le ministre intervienne en dernier ressort ${ }^{39}$. De plus, l'assemblée sera également seule habilitée à publier une Regional Housing Strategy qui fixera l'emplacement des nouveaux logements, les besoins en logements sociaux, l'investissement dans le parc existant et distribuera aux bailleurs sociaux les fonds jadis gérés par la Housing Corporation. Elle se substituera ainsi aux Government Offices et à la Housing Corporation pour identifier les besoins en nouveaux logements ainsi que leur distribution.

Comme on peut le constater, la rhétorique gouvernementale met donc l'accent sur la volonté du pouvoir central d'accroître la latitude des acteurs locaux et de favoriser les prises de décisions locales afin d'aboutir, ainsi que le formule le Livre blanc, à une 'strengthened governance in the regions' ${ }^{40}$.

\section{Deux objectifs contradictoires ?}

19 En dépit de la rhétorique officielle, tout indique qu'il y a loin du discours à la pratique. Car la volonté affichée de déléguer aux acteurs régionaux un plus grand poids décisionnel se heurte à un impératif pour le gouvernement qui est celui de faire bâtir un maximum de logements afin de mettre un terme à la crise immobilière. La politique de régionalisation en matière de logement soulève plusieurs questions et en premier lieu celle du pouvoir que possèdent véritablement les nouvelles institutions. En effet, les travaillistes n'ont jamais fait pleinement confiance au pouvoir local en ce domaine. Cette méfiance, fondée sur l'observation des décennies précédentes, s'exprime clairement dans le Livre vert de 2001, où l'on peut lire: 'There has been a tendency to avoid making the hard strategic choices, such as accomodating demand for new housing [...]. Instead a lowest common denominator is taken which in the long term can damage development in the region. ${ }^{41}$

Les travaillistes sont en effet conscients des réticences locales, en particulier des comtés et districts ruraux, à vouer des sites vierges (green fields) à la construction, en raison de l'opposition des électeurs ruraux. Ils savent que dans l'ancien système d'urbanisme et d'aménagement les comtés et districts ruraux ont toujours pesé de tout leur poids pour réduire leur part des nouveaux logements dans les directives régionales.

21 Les conflits qui ont dressé les collectivités locales contre le ministère durant les premières années de pouvoir travailliste, avant que les assemblées ne remplacent les anciennes Regional Planning Conferences, laissent sceptiques quant à la volonté de décentralisation du gouvernement. Tous ces litiges ont trouvé leur origine dans le désir de certains comtés de résister aux directives régionales du gouvernement et tous se sont soldés par la victoire du pouvoir central. L'exemple le plus frappant en a été le procès intenté par le comté du West Sussex au vice-Premier ministre John Prescott et la décision du tribunal en août 1998 d'accorder au ministre le droit d'imposer 12800 logements supplémentaires au comté lors de la révision de son plan structurel afin de respecter la directive régionale gouvernementale ${ }^{42}$. De même lors de la révision de la directive régionale des West Midlands, la même année, le gouvernement central, par le biais du Government Office régional, a contraint la région à accepter 25000 logements de plus que le chiffre suggéré par l'organisme régional d'aménagement ${ }^{43}$. Dans la région 
sud-est, là où la pénurie est la plus aiguë, mais où les associations de défense de l'environnement sont aussi les plus puissantes, le gouvernement a, là encore, remporté une victoire tout en cherchant à apaiser les passions locales. Ainsi a-t-il imposé en 2001 à la région 39000 logements par an jusqu'en 2016 dans sa directive finale ${ }^{44}$, chiffre inférieur aux 43000 logements initialement voulus par le ministre mais supérieur aux 35900 proposés par feu SERPLAN, l'ancien organisme d'aménagement du sud-est aboli en $2001^{45}$. En outre, comme vu précédemment, John Prescott a annoncé en juillet 2002 que 200000 logements supplémentaires seraient bâtis dans le sud-est, ce qui porte le total pour la région à près d'un million, chiffre en accord avec les conclusions du rapport Crow de 1999 que le gouvernement avait d'abord choisi d'abandonner face au mécontentement populaire.

\section{De la rhétorique à la pratique}

22 Ce premier bilan pouvait donc laisser craindre que les assemblées, désormais, selon la formule du Livre blanc, 'closely involved' dans la formulation des directives régionales ${ }^{46}$, disposent de peu de poids en cas de conflit avec le gouvernement central. D'ailleurs, celui-ci paraît avoir pris la précaution de soigneusement délimiter leur autonomie en la matière. Ainsi le Livre blanc précise-t-il que 'responsibility for issuing the regional spatial strategies will remain with the government ${ }^{477}$. On pouvait donc craindre que la rhétorique de décentralisation ne se résume en pratique à 'a much greater degree of joint working of Government Offices and conferences in producing Regional Planning Guidances', comme une note du DETR en 1998 semblait l'envisager ${ }^{48}$.

Ces doutes ont été confirmés par l'ingérence du ministère dans les décisions de l'assemblée régionale de l'est de l'Angleterre (East of England Regional Assembly). Celle-ci, désormais officiellement responsable de la formulation de la directive régionale, s'est prononcée en 2003 en faveur de la construction de 478000 nouveaux logements dans la région d'ici 2021. Cependant, selon les propres procès-verbaux de l'assemblée, lors d'une réunion en janvier 2004 le ministre de tutelle chargé du programme Sustainable Communities a clairement fait comprendre que le gouvernement "was disappointed that its own targets in the Sustainable Communities Plan had not been entirely met in the draft RPG14 document ${ }^{\prime 49}$ et « invitait » en conséquence l'assemblée à augmenter de 18000 logements ses prévisions.

De la même manière, les compétences des Regional Housing Boards semblent limitées. De l'aveu du conseiller chargé de la stratégie de logement de la West Midlands Regional Assembly, le Housing Board, créé en 2003 par la région afin de formuler la future stratégie régionale de logement, serait loin d'être autonome. La Housing Corporation, qui siège dans la nouvelle structure, continuerait en accord avec le ministre de décider de l'allocation des fonds et du nombre de logements sociaux dans la région et d'en contrôler le budget. Le sentiment de ce responsable est que la dynamique centrale l'emporte et que l'autonomie régionale est fortement mise en question ${ }^{50}$. Le responsable du logement pour le ODPM lui aurait même confié que l'idée maîtresse était 'consultation but no involvement of local elected members on Regional Housing Board. 51

Cette volonté d'intervenir dans le processus de décision locale pour s'assurer que les objectifs de construction gouvernementaux sont respectés tranche d'ailleurs avec la réticence du gouvernement à agir lorsque la population locale fait entendre son opposition à des projets de construction. Ainsi, il aura fallu six ans au ministre pour 
intervenir dans le conflit opposant comté du Hertfordshire et district urbain de Stevenage sur la révision du plan structurel et la construction de 10000 logements dans la ceinture verte. Ce n'est qu'en novembre 2002 que John Prescott s'est résolu à prendre en compte le mouvement local d'opposition au projet et à user de ses pouvoirs de réserve pour suspendre la révision du plan. Il semblerait donc que les assemblées régionales puissent user de leur pouvoir décisionnel seulement lorsque leurs décisions correspondent aux attentes du gouvernement. Le vernis de la responsabilité régionale paraît donc bien mince.

La décentralisation, comme elle est conçue actuellement, soulève une autre question, celle de la participation citoyenne. Car en dépit de la rhétorique officielle qui place l'accent sur des principes tels que 'choice and greater say'52 et parait inscrire les projets du gouvernement dans la 'voice option' ${ }^{\prime 53}$ il est possible de soutenir que, dans le cas du logement, la décentralisation a conduit à moins de démocratie et rendu plus difficile la participation citoyenne.

Il faut en effet garder à l'esprit que les assemblées, à la différence des anciennes conférences régionales d'aménagement décrites par le gouvernement comme 'not sufficiently inclusive ${ }^{254}$ comportent obligatoirement $1 / 3$ d'acteurs locaux non élus et donc accordent davantage de poids aux partenaires économiques dans la formulation des besoins en logement. Quand on mesure l'importance donnée à l'économie par le gouvernement dans le Livre blanc sur les régions ${ }^{55}$ et que l'on sait que la pénurie de logement est perçue par le gouvernement comme une entrave à l'emploi et à la productivitée ${ }^{56}$, il est aisé de jeter un regard cynique sur la création des assemblées. En outre, les méandres du système de partenariat que le gouvernement souhaite voir présider aux décisions en matière de logement sont tels qu'il est parfois nécessaire de nommer spécialement un conseiller au logement, comme l'a fait la West Midlands Regional Assembly, pour expliquer la nouvelle donne. Nombre d'élus locaux sont déroutés par le nouveau système et ont paradoxalement le sentiment d'être exclus du processus décisonnel ${ }^{57}$. Cette impression a été confirmée par un bilan réalisé par le Chartered Institute of Housing dans son évaluation des stratégies régionales de logement en septembre 2003 pour le compte de John Prescott, qui concluait au manque de clarté des structures, liens et responsabilités des organismes désormais impliqués ${ }^{58}$. On peut donc craindre que le nouveau processus ne favorise ni la transparence ni la participation citoyenne, objectifs majeurs du programme de décentralisation. De plus, le partenariat censé présider à la formulation des choix en matière de logement ne laisse de place qu'à des groupes institutionnalisés, tout comme l'ancien système.

Enfin, certaines réformes présentées comme allant dans le sens de la décentralisation et renforçant l'échelon régional semblent démentir la rhétorique participative. C'est la raison pour laquelle de nombreuses associations soupçonnent le gouvernement, très souvent confronté à l'opposition de riverains (nimbies ${ }^{59}$ ), de vouloir passer en force. Ainsi, la réforme du planning system qui prévoit le remplacement des directives régionales par des stratégies régionales spatiales impliquerait l'abolition des plans structurels des comtés ${ }^{60}$ et donc du système d'objection et d'enquête publique (examination in public) au niveau de ces mêmes comtés, système présenté par le gouvernement comme 'time consuming and adversarial' ${ }^{61}$. Comme la Local Government Association, la Town and Country Planning Association et le CPRE l'ont souligné, cela contribuerait à réduire les possibilités offertes à la population de s'exprimer et à créer un vide entre l'échelon régional et local. Cette même réforme, paradoxalement, 
prévoyait également de retirer aux régions le pouvoir de débattre de certains grands projets nationaux (Major Infrastructure Projects) dont le principe aurait été approuvé par le parlement seul ${ }^{62}$. Enfin, tout aussi surprenant, le gouvernement projetait de confier le pilotage des quatre zones de croissance décidées par le pouvoir central à des Urban Developement Corporations. Ces organismes, utilisés sous les conservateurs dans les années 1980 dans le cadre de la rénovation urbaine, laissent peu de place à la participation citoyenne. Ils sont en effet créés par ordonnance ministérielle, sont uniquement responsables devant le gouvernement central, sont dispensés d'enquête publique et gèrent seuls les permis de construire dans le périmètre de leur zone d'action. Face à la levée de boucliers déclenchée par le projet de loi, le gouvernement a finalement retiré les deux premières dispositions du texte final du Planning and Compulsory Purchase Act voté en 2004.

\section{Conclusion}

Le chantier de la régionalisation et celui du logement se rencontrent donc en un point hautement sensible, qui est celui du calcul des besoins régionaux en logements. Pris entre des intérêts contradictoires, le gouvernement semble vouloir recourir à la centralisation et chercher à imposer la ligne définie par le pouvoir central. Le comportement du ministère paraît donc démentir la rhétorique officielle sur l'importance de la gouvernance locale. Pour l'heure, bien que le gouvernement ait cédé face à la critique sur certains points, priorité est donnée à la construction de logements et l'objectif de renforcer la croissance par une offre suffisante en logements semble prévaloir sur le programme de régionalisation. Le pouvoir central semble avoir du mal à accepter qu'un programme de gouvernement régional implique de transférer des pouvoirs aux régions mais aussi d'en retirer au centre.

Le transfert de véritables pouvoirs en matière d'aménagement et de logement en particulier devra probablement attendre l'élection d'organismes démocratiquement élus avec un mandat populaire. On pourra alors juger de la sincérité du New Labour et de sa volonté de confier aux élus du peuple la maîtrise des politiques de logement au niveau régional, comme le Livre blanc de 2002 consacré aux régions l'a promis. Le rejet $\mathrm{du}$ projet du gouvernement par les habitants du nord-est de l'Angleterre lors du premier référendum sur la question, le 4 novembre 2004, laisse penser que les dispositions annoncées en 2002 dans le Livre blanc et confirmées en 2004 par le vote du Planning and Compulsory Purchase Act marqueront longtemps les limites de la décentralisation dans le cas du logement.

\section{BIBLIOGRAPHIE}

BALCHIN, Paul. Housing Policy. London: Routledge, 1996, 312 p.

BLAIR, Tony. New Britain : My Vision of a Young Country. London: $4^{\text {th }}$ Estate, 1996, 338 p. 
BURNS, Dany et al. The Politics of Decentralisation : Revitalising Local Democracy. London: Macmillan, 1994, $304 \mathrm{p}$.

DEPARTMENT OF ENVIRONMENT. Housing Policy: A Consultative Document. Cmnd. 6851. London: HMSO, 1977, $154 \mathrm{p}$.

DEPARTMENT OF THE ENVIRONMENT. Projections of Households in England to 2016. London: HMSO, $1995,99 \mathrm{p}$.

DEPARTMENT OF ENVIRONMENT, TRANSPORT AND THE REGIONS. The Future of Regional Planning Guidances. London: The Stationery Office, 1998, 26 p.

DEPARTMENT OF ENVIRONMENT, TRANSPORT AND THE REGIONS. Regional Planning, PPG11. London: DETR, 1999, 83 p.

DEPARTMENT OF ENVIRONMENT, TRANSPORT AND THE REGIONS. Quality and Choice : A Decent Home for All. London: The Stationery Office, 2000, $132 \mathrm{p}$.

HER MAJESTY'S TREASURY. The Barker Review : The Final Report. London: The Stationery Office, $2004,157 \mathrm{p}$.

NEW LABOUR. Because Britain deserves Better. London: The Labour Party, 1997, 40 p.

NEW LABOUR. Ambitions for Britain. London: The Labour Party, 2001, 44 p.

OFFICE OF THE DEPUTY PRIME MINISTER. Planning : Delivering a Fundamental Change. London: The Stationery Office, $2001,56 \mathrm{p}$.

OFFICE OF THE DEPUTY PRIME MINISTER. Your Region, Your Choice. Cmnd. 5511. London: The Stationery Office, $2002,110 \mathrm{p}$.

OFFICE OF THE DEPUTY PRIME MINISTER. Sustainable Communities : Building for the Future. London: The Stationery Office, 2003, $68 \mathrm{p}$.

VIGAR, Geoff et al. Planning, Governance and Spatial Strategy in Britain. London: Routledge, 2000, $314 \mathrm{p}$.

\section{NOTES}

1. New Labour, Because Britain Deserves Better, London: The Labour Party, 1997, p. 34-35.

2. Department of the Environment, Housing Policy: A Consultative Document, Cmnd. 6851, London: HMSO, 1977. Celui-ci recommandait déjà de bâtir 310000 logements par an jusqu'en 2000, voir Paul BALCHIN, Housing Policy, London: Routledge, 1996, p. 36.

3. Departement of Environment, Transport and the Regions, Quality and Choice: A decent Home for all, London: The Stationery Office, 2000.

4. La Housing Research Foundation par exemple, évaluait en 1984 le nombre de logements supplémentaires nécessaires à 3 millions d'ici 2000 soit 187500 par an. La Builders' Employers Federation a estimé en 1985 qu'il y avait un déficit annuel de 100000 nouveaux logements. La School of Advanced Urban Studies en 1987, en réponse à la English Condition Survey de 1981 qui avait démontré l'existence de 2 millions de logements insalubres, recommandait 162000 par an, voir Paul BALCHIN, op.cit., p. 36.

5. Voir Paul BALCHIN, op.cit., p. 17. Son montant brut passant de 12,5 à 4,9 milliards de livres entre 1979 et 1995 selon The Economist, 'Social Housing : A Conundrum', 17/08/96, p. 28.

6. Department of the Environment, Housing and Construction Statistics, 1996. 
7. Department of the Environment, Projections of Households in England to 2016, London: HMSO, 1995, p. 1.

8. 'So we will lift the ban on the use of these funds and allow their release on a phased basis so that councils can start to build and reverse the neglect of recent years. The effect would be to provide more housing [...] and to increase investment.' Tony BLAIR, New Britain: My Vision of a Young Country, London: $4^{\text {th }}$ Estate, 1996, p. 196

9. The Labour Party, 1997, op.cit., p. 26.

10. De nouveaux programmes urbains tels que New Deal for Communities et Neighbourhood Renewal Fund ont ainsi été créés avec à la clef $£ 1.9$ milliards et 900 millions de livres respectivement. En outre, 1 milliard de livres de déductions fiscales a été offert afin de convaincre le secteur privé d'investir dans les zones urbaines, dans le logement en particulier. Enfin, 250 millions de livres depuis fin 2000 sont proposés à 10000 employés des services publics dans le cadre du programme Starter Home Initiative afin de leur permettre d'acheter leur premier logement. Voir New Labour, Ambitions for Britain, London: The Labour Party, 2001, p. 14, p. 29.

11. 'Building boom, but where are the houses?', The Guardian, 5/10/03.

12. Sur le long terme le déclin est plus visible : en 2001, 179000 logements ont été bâtis, dont 154000 par le privé, alors qu'au plus fort de la croissance, en 1968, le chiffre total était de 426000 , voir Office of National Statistics, Social Trends, London: The Stationery Office, 2002, p. 165.

13. Office of National Statistics, Social Trends, London: The Stationery Office, 2003, p. 179.

14. 'Associations should borrow to build', The Guardian, 10/12/03.

15. HM Treasury, The Barker Review : Final Report, London: The Stationery Office, 2004, p. 125.

16. Les zones de croissance ont été annoncées par John Prescott le 18 juillet 2002 avant que le projet de communauté ne soit présenté devant le parlement en février 2003.

17. 'Labour will reform the planning system to speed up decision-making, promote the most efficient use of land and strike the right balance of environmental protection, safer communities, and economic growth.' New Labour, 2001 Manifesto, op.cit., p. 14.

18. 'Reform of the planning system is the key to proper used of land and enabling well designed housing developments to happen.' Voir Office of the Deputy Prime Minister, Sustainable Communities, London: Stationery Office, p. 30.

19. Depuis la réforme du gouvernement local de 1972 et l'abolition des comtés métropolitains en 1986 ainsi que la réorganisation partielle dans les années 1990 par les conservateurs, celles-ci incluent les county councils et les district councils, les metropolitan districts et unitary local authorities.

20. Local plan, structure plan, ou unitary development plan, soumis à révision tous les 5 ans.

21. Office of the Deputy Prime Minister, Planning: Delivering a Fundamental Change, London: the Stationery Office, 2001, § 4.8-4.14.

22. 'Prescott gets tough on housing', The Guardian, 9/4/2003.

23. 'It gives people living in the English Regions the chance to have a greater say over the key issues that affect them.' ODPM, Your Region, Your Choice, Cmnd. 5511, London: The Stationery Office, 2002, Préface.

24. 'To devise tailored regional solutions to regional problems', ibid. Cf. aussi § 3,12 et 1.13.

25. Après le vote du Regional Development Agencies Act en 1998.

26. 'To take a strategic lead in driving improved economic performance in the English Regions.' ODPM, Your Region, Your Choice, op.cit., § 2.5

27. Les RDA tirent leur financement $\mathrm{du}$ budget des quangos qu'elles remplacent (English Partnerships, Rural Development Corporation, SRB) et d'une partie de la plus-value de la vente des HLM depuis 1979.

28. 'They work closely with regional partners and local people to maximise competitiveness and prosperity in the regions and to promote social inclusion.' ODPM, Your Region, Your Choice, op.cit., \$2.28. Ils sont financés et pourvus en personnel par les neuf ministères mécènes. 
29. 'In time we will introduce legislation to allow the people, region by region to decide in a referendum whether they want directly elected regional government. Only where clear regional popular consent is established will arrangements be made for elected regional assemblies', 1997 Manifesto, op.cit., pp. 34-35. 30. DETR, The Future of Regional Planning Guidances, London: The Stationery Office, 1998, § 5.3.

31. Ibid., § 2.8 .

32. Les projections démographiques nationales et sous-nationales publiées tous les trois ans en utilisant la cohort survival method qui consiste à appliquer des taux de mortalité, de fertilité et de migration à une population de base, étaient converties par le DOE en estimations du nombre de foyers et réparties entre collectivités locales, comtés, districts métropolitains et boroughs londoniens, en fonction des mouvements migratoires

33. En prenant en compte le nombre de logements existants, le pourcentage de logements vides, l'état du parc, le pourcentage de terrains industriels à recycler, et les contraintes environnementales.

34. Cette directive guidait les comtés dans l'élaboration de leur plan structurel, plan qui distribue les besoins en logements évalués par l'État central entre les districts après enquête publique (Examination in Public). Enfin, l'emplacement de ces logements est défini lors de la réunion publique du district planning committee (Local Plan Inquiry) qui précède la formulation du plan local. 35. Par le biais de directives nationales sur l'aménagement du territoire, de la consultation entreprise avant la publication de la version définitive des directives régionales et en dernier lieu de l'usage de pouvoirs de réserve pour imposer la volonté centrale à la périphérie si les plans des collectivités locales étaient jugés incompatibles avec les RPG.

36. DETR, The Future of RPG, op.cit., § 5.5.

37. Ébauche soumise à un panel nommé par le Secretary of State pour enquête publique désormais statutaire (Public Examination). Les conclusions de cette enquête sont ensuite rapportées au SoS. Il publie alors un rapport sur les conclusions du panel expliquant les modifications éventuelles qu'il désire apporter. S'il y a modifications, s'en suit une période de consultation puis le RPG final est publié par le SoS. Voir DETR, PPG11, Regional Planning, London: DETR, 2000, pp. 9-27.

38. ODPM, Sustainable Communities : Building for the Future, London: The Stationery Office, 2003, $\S$ 6.11.

39. ODPM, Your Region, Your Choice, op.cit., § 4.34.

40. Ibid., § 2.28 .

41. ODPM, Planning: Delivering a Fundamental Change, op.cit., § 4.41 et $\S 4.45$.

42. Celle-ci imposait également 16400 logements de plus au Somerset et 6000 de plus au Norfolk que les élus ne le souhaitaient, voir 'Dismay over rural housing ruling', The Independent, 2/8/98.

43. Geoff VIGAR et al., Planning, Governance and Spatial Strategy in Britain, London: Routledge, 2000, p. 101.

44. Campaign to Protect Rural England, Press Release, 13.3.03.

45. Bucks County Council, Strategic Planning Service, County Council Welcomes Unchanged Housing Proposals, March 2001.

46. ODPM, Your Region, Your Choice, op.cit., § 2.23.

47. Ibid., § 2.19 .

48. DETR, The Future of RPG, op.cit., § 5.3.

49. East of England Regional Assembly, Draft Regional Planning Guidance for the East of England (RPG14), 5/2/2004, § 2.3 .

50. Propos recueillis lors d'entretiens réalisés en février 2004 et octobre 2004 à Birmingham avec le Housing Strategic Adviser de la WMRA.

51. Ibid.

52. ODPM, Your Region, Your Choice, op.cit., Introduction. 
53. Modèle de A.O. HIRSCHMAN exposé dans Exit, Voice and Loyalty, Cambridge, Mass.: HUP, 1970 et repris par la Urban Studies School de Bristol pour être appliqué à la Grande-Bretagne dans Dany BURNS et al., The Politics of Decentralisation, London: Macmillan, 1994.

54. ODPM., Your Region, Your Choice, op.cit., § 2.20.

55. Ibid., voir Overview ( $\$ 3$ ) ou chapitre 1 : Understanding the Regional Dimension.

56. Ibid., § 4.9 : 'A region's needs in respect of housing and transport infrastructure depend partly on its strategy for economic development, but its economic strategy also needs to take account of priorities arising from its housing [...] and spatial strategies' et $\$ 4.32$ : 'effective planning can promote economic prosperity by delivering land for development in the right place and at the right time'.

57. 'Local councillors do not understand how it works and feel less involved. They are trying to understand where the point of decision is', propos recueillis lors des entretiens cités ci-dessus.

58. 'There is not always clarity concerning the structures, relationships and responsibilities of the different bodies involved in the process', Chartered Institute of Housing, An Evaluation of Regional Housing Strategies, London: CHI, 2003, p. 2.

59. Acronyme de : 'Not In My Back Yard'.

60. 'We believe that the county no longer remains the most appropriate level at which to consider many of the key strategic planning issues’, ODPM, Planning : Delivering a Fundamental Change, op.cit., § 4.37.

61. Ibid., $\$ 4.26$

62. L'accord de la collectivité locale n'aurait plus été nécessaire mais un simple vote parlementaire aurait suffi, suivi d'une enquête publique pour les détails, voir ODPM, Planning: Delivering a Fundamental Change, op.cit., § 6.4.

\section{RÉSUMÉS}

Le gouvernement de Tony Blair mène de front de multiples réformes depuis son élection en 1997. En particulier, il s'efforce de mettre un terme à la pénurie de logements qui menace l'économie et la cohésion sociale du pays, ce qui implique un fort dirigisme du gouvernement central. D'autre part, il s'emploie à mettre en œuvre un vaste programme de décentralisation, qui passe par une plus grande autonomie des régions. Cet article tente de montrer en quoi ces deux projets s'avèrent difficiles à concilier et en quoi ils mettent à mal certains des principes affichés du New Labour.

The Blair government has been battling on many fronts since it was elected in 1997. In particular it has been striving to put an end to the housing shortage which is threatening the economy of the country as well as social cohesion. This requires substantial central government intervention. It has also been attempting to implement a vast program of decentralisation which entails greater regional autonomy. This article seeks to demonstrate why these two policies are difficult to reconcile and how they contradict certain official New Labour principles.

\section{AUTEUR}

\section{DAVID FÉE}

Université de Paris III 